\title{
The Modularity and Dynamicity of miRNA-mRNA Interactions in High-Grade Serous Ovarian Carcinomas and the Prognostic Implication
}

\author{
Wensheng Zhang ${ }^{1}$ Andrea Edwards ${ }^{1}$, Wei Fan ${ }^{2}$, Erik K. Flemington ${ }^{3}$, Kun Zhang ${ }^{1 \S}$ \\ ${ }^{1}$ Department of Computer Science, Xavier University of Louisiana, 1 Drexel Drive, New Orleans \\ LA 70125 \\ ${ }^{2}$ Big Data Lab, Baidu Research, 1195 Bordeaux Dr., Sunnyvale, CA 94089 \\ ${ }^{3}$ Tulane Health Sciences Center, Tulane Cancer Center, Tulane University, 1700 Tulane Ave, \\ New Orleans, LA 70112 \\ $\S$ Corresponding author \\ Email addresses:
}

WZ: wzhang@xula.edu

AE: aedwards@xula.edu

WF: fanwei03@baidu.com

EKF: eflemin@tulane.edu

KZ: kzhang@xula.edu 


\begin{abstract}
Ovarian carcinoma is the fifth-leading cause of cancer death among women in the United States. Major reasons for this persistent mortality include the poor understanding of the underlying biology and a lack of reliable biomarkers. Previous studies have shown that aberrantly expressed MicroRNAs (miRNAs) are involved in carcinogenesis and tumor progression by posttranscriptionally regulating gene expression. However, the interference of miRNAs in tumorigenesis is quite complicated and far from being fully understood. In this work, by an integrative analysis of mRNA expression, miRNA expression and clinical data published by The Cancer Genome Atlas (TCGA), we studied the modularity and dynamicity of miRNA-mRNA interactions and the prognostic implications in high-grade serous ovarian carcinomas. With the top transcriptional correlations (Bonferroni-adjusted p-value < 0.01) as inputs, we identified five miRNA-mRNA module pairs (MPs), each of which included one positive-connection (correlation) module and one negative-connection (correlation) module. The number of miRNAs or mRNAs in each module varied from 3 to 7 or from 2 to 873. Among the four major negativeconnection modules, three fit well with the widely accepted miRNA-mediated posttranscriptional regulation theory. These modules were enriched with the genes relevant to cell cycle and immune response. Moreover, we proposed two novel algorithms to reveal the group or sample specific dynamic regulations between these two RNA classes. The obtained miRNAmRNA dynamic network contains 3350 interactions captured across different cancer progression stages or tumor grades. We found that those dynamic interactions tended to concentrate on a few miRNAs (e.g. miRNA-936), and were more likely present on the miRNA-mRNA pairs outside the discovered modules. In addition, we also pinpointed a robust prognostic signature consisting of 56 modular protein-coding genes, whose co-expression patterns were predictive for the survival time of ovarian cancer patients in multiple independent cohorts.
\end{abstract}

Key words: ovarian cancer, mRNAs, miRNAs, network, modules, dynamic interactions, prognostic signature.

\title{
1. Introduction
}

Ovarian cancer is the fifth-leading cause of cancer death among women in the United States (Siegel et al., 2014). The disease is often called a "silent killer" since its occurrence is usually not detected until an advanced stage. About $70 \%$ of the deaths occur in patients with advancedstage, high-grade serous ovarian carcinomas. The mortality rate has not improved significantly in the past three decades (Bast et al., 2009). Except for the detection delay and inaccessible location of the ovaries, major factors accounting for the persistent mortality include the poor understanding of the underlying biology and a lack of reliable biomarkers (Li et al., 2012). As a result, there is a pressing need for the investigation of the mechanisms underlying the development of this disease as well as the identification of robust early biomarkers for diagnosis and prognosis.

MicroRNAs (miRNAs) are short ( 22nt) non-coding RNAs derived from genome-encoded stem loop precursors. As the crucial post-transcriptional regulators of gene expression in metazoans, miRNAs primarily bind to the 3' UTR sequences of messenger RNAs (mRNAs), usually resulting in translational repression or mRNA degradation (Filipowicz et al., 2008; Bartel, 2009). About 2000 human miRNAs have been discovered so far, and these miRNAs have collectively been implicated in a wide range of key biological processes, including cell growth, differentiation and proliferation, embryonic development, and apoptosis. Growing evidence has 
shown that aberrantly expressed miRNAs are likely to contribute to cancerous lesions (Aguda et al., 2008; Visone and Croce, 2009; Wang et al., 2010; Dvinge et al., 2013). Therefore, the use of miRNAs as potential diagnostic tools and therapies has been suggested for the treatment of cancer. Nevertheless, the interference of miRNAs with carcinogenesis is quite complicated and far from being fully understood. A systems-level analysis of the miRNA-mediated regulation in cancer development and progression would enable us to better decipher the roles of miRNAs in tumorigenesis (Bandyopadhyay et al., 2010; O'Day and Lal, 2010; Sumazin et al., 2011; Bisognin et al., 2012).

Modularity is a basic characteristic of various biological networks (Newman, 2006; Schwarz et al., 2008; Barabasi et al., 2011). Networks with high modularity have dense connections between the nodes within modules but sparse connections between nodes in different modules. To date, a number of methods have been developed to infer miRNA-mRNA regulation (or correlation) modules using the genome-wide transcription and sequence affinity information (Joung et al., 2007; Tran et al., 2008; Michoel et al., 2009; Peng et al., 2009; Jayaswal et al., 2011). In a previous study (Zhang et al., 2012a), we applied a clustering-based algorithm to the miRNA and mRNA gene expression data of human prostate cancer cells, finding highly-informative modules specific for primary tumors (PPCs). On the other hand, the transcriptional relationship of a miRNA-mRNA pair can be dynamic. That is, the interaction status and strength/direction between these two molecules may vary even with the progression of the same cancer case. For example, in (Zhang et al., 2012a), we found that the modules discovered for PPCs rarely overlapped with those for metastatic prostate cancers (MPCs). Therefore, it would be interesting to explore, as the cancer progresses, in which manner and to what extent the changes in miRNA expression impact gene expression, biological pathways and networks as well as patient prognosis.

In this paper, we primarily studied the modularity and dynamicity of miRNA-mRNA interactions in high-grade serous ovarian carcinomas (Ov-HGSCs) with respect to different cancer progression stages or tumor grades. The research was based on an integrative analysis of mRNA expression, miRNA expression and clinical data released by TCGA. Similar to (Zhang et al., 2012a), a clustering-based method was applied to module discovery. Two new algorithms, namely a regression model and a statistical approach driven by nonparametric methods, were proposed for (sample) group and case focused dynamics study. We further investigated the prognostic implications of the major results by identifying a robust prognostic signature. The coexpression patterns of the genes contained in the signature were predictive for the survival time of ovarian cancer patients in multiple independent cohorts. Fig. 1 summarizes the scheme of the study flow, and the details of each step are described in Sections 2 and 4.

\section{Results}

For ease of presentation, we use the term "miRNA-mRNA interaction" hereafter to represent different context-specific interaction measures studied in this work, including correlation, regression or "interaction strength". The common biological mechanism underlying these measurements is the mutual regulation between miRNAs and mRNAs or the regulation of both molecules by the common upstream regulators.

In inferring the miRNA-mRNA interaction network from TCGA data, we focused on 11684 well annotated mRNA genes and 86 pre-selected cancer relevant miRNAs (see Section 4). The transcriptional correlations (connections) between miRNAs and mRNAs were calculated by the 
Pearson coefficient (r) and the significance levels were evaluated by a t-test (using the function t.test in the R package "stats") in which the null hypothesis was $H_{0}: r=0$. In the modularity study, we concentrated on a condensed miRNA-mRNA network with $\sim 18 \mathrm{~K}$ top connections (Bonferroni-corrected p-value $<0.01$ ). An enlarged relationship set (S0), containing $\sim 271 \mathrm{~K}$ top connections (ordinary p-value $<0.01$ ), was analyzed in the dynamicity study.

\subsection{Modularity}

Using the condensed network as the input, we identified the miRNA-mRNA modules by a clustering-based algorithm. Within an individual module, each miRNA or mRNA has at least two connections to the corresponding modular mRNAs or miRNAs. As shown in Table 1 and Fig. 2, we pinpointed five miRNA-mRNA module pairs (MPs). Each MP includes one positiveconnection (correlation) module and one negative-connection (correlation) module. The number of miRNAs or mRNAs in each module varies from 3 to 7 or from 2 to 873. Most of the modules contain the sequence-specific DNA binding transcription factor (TF) genes that may take a role as the mediators for the miRNA-mRNA connections. The two members (such as modu-1-ps and modu-1-ne) of each MP contain the same or related miRNAs but different mRNAs. Two modules of distinct MPs (such as modu-1-ps and modu-2-ps) consist of different miRNAs and varied (or partially overlapped) mRNA sets. Within a positive or negative-connection module (denoted by a "-ps" or "-ne" extension in the IDs), the transcriptional correlations of the involved miRNAs and mRNAs are consistently positive or negative. Regardless of the connection type, the mRNA set in each module largely imitates a co-expressed gene cluster. Among the four major negative-connection modules, three fit well with the popularly recognized miRNAmediated post-transcriptional regulation theory. That is, the $3^{\prime} \mathrm{UTR}$ sequences of the involved mRNAs are enriched with the target site motifs of the modular miRNAs (Fig. 3). As with [1], we call them semi-canonical regulatory modules (semi-CRM).

We performed functional enrichment analysis on the modular genes using the David tool (Huang da et al., 2007). As presented in Table 1, nearly all the top over-represented gene ontology (GO) terms are directly related to three cancer relevant functional hallmarks (Hanahan and Weinberg, 2011), i.e. extracellular matrix (EM), immune response (IR) or cell cycle (CCY). We further summarized the implications of the enrichment profiles as follows. First, miRNA-106b/-107/148b/-93 and miRNA-17/-18a/-18b/-20a/-20b represented the miRNA subsets regulated by the same (or similar) up-stream regulators playing similar biological roles. Their expression levels were negatively (or positively) correlated with the IR (or CCY) genes. Second, contrary to miRNA-17/-18a/-18b/-20a/-20b, miRNA-222/-29a/-29c/-let-7b positively interacted with IR genes but negatively interacted with CCY genes. Lastly, miRNA-143/-145/-214 and miRNA$140-3 \mathrm{p} /-146 \mathrm{a} /-150 /-155 /-21 /-223 /-224$ were less likely to be the direct regulators for the cognate modular genes in Ov-HGSCs. This was because their connections with those genes were predominated by positive transcriptional correlations, and the 3' UTRs of those genes, as a whole, didn't contain more sequence affinity-based miRNA binding sites than that of an average human gene.

\subsection{Dynamicity}

To reveal the dynamic patterns that were both statistically significant and biologically relevant, we proposed two methods to tackle this problem. One is a regression model and the other is a statistical approach driven by nonparametric tests. The former is more focused on capturing 
patterns with (sample) group effects while the latter is more specific to each individual sample case.

Heterogeneous regression slopes In the regression model, we treated the expression measure of mRNAs as the dependent variable (y) and the expression of miRNAs as a predictive variable (x) (See Section 4 for details). The purpose was to identify the miRNA-mRNA pairs which demonstrated cancer development-dependent interaction patterns being indicated by heterogeneous regression slopes in different groups of cancer samples as to cancer progression. For each of the miRNA-mRNA pairs, we ran the regression analysis twice.

In the first run, the ovarian carcinomas were partitioned into stage-II, -III, and -IV groups. Each group accounted for $4.5 \%, 79.2 \%$ and $16.3 \%$ of the entire set, respectively. We determined the interaction dynamicity by a set of statistical tests with $H_{0}: \gamma_{1}=\gamma_{2}=\gamma_{3}$ being the general null hypothesis, where $\gamma_{1}, \gamma_{2}$ and $\gamma_{3}$ are the regression coefficients of $\mathrm{y}$ on $\mathrm{x}$ (see the previous paragraph) for the three cancer groups. A small p-value calculated in these tests was regarded as the evidence for dynamicity. Using 0.00023 as a p-value cutoff (corresponding to FDR < 0.2 ) for the comparison between $\gamma_{2}$ and $\gamma_{3}$, we identified 327 dynamic miRNA-mRNA pairs consisting of 70 miRNAs and 220 coding genes. For example, Fig. 4 illustrates the progression-stage relevant regression of the expression of gene FOXF1 on the expression level of miR-17 or let-7g. The most frequent miRNAs were miRNA-127-5p (with 43 connections) and miRNA-126 (with 33 connections). Two cancer relevant gene ontology terms GO:0007155 cell adhesion and GO:0031012 extracellular matrix were over-represented (BH adjusted $\mathrm{p}<0.01$ ) by the member genes. We didn't report the comparisons between $\gamma 1$ and $\gamma 2(\gamma 3)$ as the results were not considered meaningful according to the criterion of FDR $<0.2$. The statistical insignificance between the stage-II and stage-III (IV) groups in terms of the regression measure was likely due to the small size $(\mathrm{n}=13)$ of the stage-II set.

In the second run, 474 cancer samples were divided into grade- 2 and -3 groups. Each group accounted for $12 \%$ and $88 \%$ of the entire set, respectively. Based on a similar hypothesis test and the same FDR cutoff employed in the first run, we identified 77 dynamic miRNA-mRNA pairs. Fig. S1 showcased the tumor-grade relevant regression of the expression of gene CD70 on the expression level of miR-622 or let-7e. The genes MAB21L2 and TNFRSF10C are predominant in these dynamic relationships. They have 28 and 20 connections with the involved 48 miRNAs, respectively. TNFRSF10C is a potential tumor suppressor gene. Its inactivation by chromosomal deletion and promoter methylation may play an important role in prostate cancer development (Cheng et al., 2009). To the best of our knowledge, the involvement of proteins coded by MAB21L2 in carcinogenesis has rarely been reported yet. However, a recent study on mice showed that the deletion of this gene can cause decreased cell proliferation and increased apoptosis in embryonic heart and liver (Saito et al., 2012).

Heterogeneous interaction strength In our second attempt to study the dynamic miRNA-mRNA interactions, a statistical analysis driven by nonparametric methods was performed to identify the miRNA-mRNA pairs which showed divergent "interaction strength (IS)" in different sample groups regarding cancer progression. IS was calculated for individual carcinomas by an expression rank transition-based algorithm (see Section 4). A large positive (or negative) IS value may indicate a strong sample-specific positive (or negative) regulation between a miRNA and an mRNA. This value can also measure the co-regulation of these two molecules by a common upstream regulator. The assumption underlying the method is that, the interaction between a miRNA and an mRNA gene may vary across individual tumors and depend on the 
progression status of cancer. Similar to the regression analysis, we conducted the nonparametric study twice.

In the first run, we split the ovarian carcinomas into stage-II, -III, and -IV groups. In particular, two stage-IIA tumors, the "youngest" tumors in the focused TCGA cohort, were treated as the base-line samples and were excluded from the stage-II group. For each miRNA-mRNA pair, we evaluated the differences of the derived interaction strength variable IS between the three groups by a Kruskal-Wallis test (using the function kruskal.test in the R package "stats") in which the null hypothesis stated that the mean ranks of these groups were the same. With 0.00062 as a pvalue cutoff (corresponding to FDR < 0.1), we identified 1236 miRNA-mRNA pairs in which 67 miRNAs and 794 coding genes were involved. Take the genes involved in the KEGG pathway $\sim$ ECM-receptor-interaction as an example, Fig. 5 illustrates the miRNA-mRNA interaction strengths over different (cancer) progression stages. The most frequent miRNAs included miRNA-145, -19a, -143 and -221, and each of them had 327, 161, 95 and 94 connections with the involved mRNA genes, respectively. Two cancer-relevant gene ontology terms GO:0010941 regulation of cell death and GO:0031012 extracellular matrix, as well as the KEGG pathway NOD-like receptor signaling (Fig. S2), were over-represented (BH adjusted p < 0.02 ) by the mRNA genes.

In the second run, we partitioned the 474 cancer samples into grade- 2 and -3 groups. For each miRNA-mRNA pair, we evaluated the differences of the derived interaction strength variable IS between the two groups by a Mann-Whitney test(using the function wilcox.test in the R package "stats") in which the null hypothesis stated that the mean ranks of the groups were the same. With 0.00022 as a p-value cutoff (corresponding to FDR < 0.1), we identified 1750 miRNAmRNA pairs. Take the genes annotated to the Gene Ontology term cell-cycle-pathway as an example, Fig. S3 illustrated the miRNA-mRNA interaction strengths across different tumor grades. 1115 coding mRNA genes, by which KEGG pathways ECM-receptor interaction and cell cycle were over-represented (BH adjusted $\mathrm{p}<0.06$ ), are involved in these miRNA-mRNA pairs. The most frequent miRNAs are miRNA-936, -622, -34a, -671-5p and -1224-5p. They have 608, 241, 238,120 and 110 connections with the involved mRNA genes, respectively.

\subsection{Comparison of modular and dynamic miRNA-mRNA interactions}

By separately combining the results obtained from the modularity and dynamicity studies, we generated two miRNA-mRNA interaction subsets, S1 (summarized in Table S1) and S2 (summarized in Table S2). S1 contained 10812 modular connections in the ten modules summarized in Table 1. S2 contained 3350 dynamic relationships identified by the regression model and/or the nonparametric analysis. As shown in Fig. 6A, S1 and S2 are two subsets of the larger relationship set (S0). The intersection of S2 and S1 accounts for $0.6 \%$ of S1. This

percentage was significantly lower (binomial test $\mathrm{p}=2.32 \mathrm{x}^{-10}$ ) than the proportion $(1.2 \%)$ of S2 in S0. This indicates that a dynamic interaction tends to be present in the miRNA-mRNA pairs outside the modules with strong correlations.

We further scrutinized the identified interactions in S1 and S2 by looking into the distribution profiles of the connections with respect to the involved miRNAs. As shown in Fig. 6C, most connections in $\mathrm{S} 2$ concentrated on several miRNAs, especially on miRNA-936 which linked to 613 mRNA genes in total. This is quite different from the scenario in the modular interaction subset S1 (Fig. 6B), where the connections more evenly distributed over the involved miRNAs. Among the ten most frequent miRNAs in S2, only miRNA-19a has more sequence affinity based 
target sites on the connected genes compared to an average gene (Fisher's test $\mathrm{p}=0.05$ ). MiRNA-145, a documented transcriptional signature for cancer progression (Peng et al., 2011; Yin et al., 2013), was the second most frequent miRNA in S2.

\subsection{Prognostic implication}

We investigated the prognostic implication of miRNA-mRNA interactions by a comprehensive multi-step approach. First, using a Cox Hazard regression model and the TCGA data, we identified 333 mRNA genes with the expression levels being predictive $(\mathrm{p}<0.01)$ for the overall survival time (OS) of ovarian cancer patients. Then, we extracted three subsets from these genes.

G1 $(n=56)$ : the predictive genes that are contained in the modular miRNA-mRNA interaction subset $\mathrm{S} 1$ but not in the dynamic miRNA-mRNA interaction subset $\mathrm{S} 2$.

$\mathrm{G} 2(\mathrm{n}=55)$ : the predictive genes that are contained in $\mathrm{S} 2$ but not in $\mathrm{S} 1$.

G3 $(n=194)$ : the predictive genes that are in neither $S 1$ nor $S 2$.

Finally, we tested if these three gene sets could be individually used as a macro prognostic signature. The evaluation was performed on the datasets of TCGA and another five independent cohorts of serous ovarian carcinomas.

As shown in Fig. 7, the G1 genes (Table S3) constitute a robust macro signature for the survival prediction of the cancer patients. Based on the signs (+ or -) of the first or second principal component (PC) scores calculated from the Expression Matrix of the Signature Genes (EMSG) using the singular value decomposition (SVD) algorithm, the cancer patients were split into two groups with good or poor clinical outcomes. Except for the Crijns cohort (Crijns et al., 2009), the difference between these two groups was significant as the p-values of both the log-rank test and Cox-PH regression were less than 0.05 . In contrast, the signatures constituted by the $\mathrm{G} 2$ genes or G3 genes carried less predictive power (Fig. S4). We further noted that which PC(s) of the EMSG could serve as the robust macro prediction variable for a specific dataset was somewhat related to the choice of microarray platform. Specifically, for the cohorts of Bonome (Bonome et al., 2008) and Tothill (Tothill et al., 2008), where the gene expression were measured on a Affymetrix platform, the robust macro prediction variable was the second PC of the EMSG; However, for the other three validation cohorts, where a non-Affymetrix platform was used for gene expression profiling, the robust macro prediction variable was the first PC of the EMSG; For the training (TCGA) cohort with the expression data being generated by these two types of platforms, both the first and the second PCs were the significant predictive variables.

\section{Discussion}

In this study, we employed a clustering-based algorithm to identify miRNA-mRNA interaction (correlation) network modules in high-grade serous ovarian carcinomas. The method is similar to that used in our previous study of prostate cancer (Zhang et al., 2012a). Looking into these two studies, we find that the module modu-1-ps identified in this work resembles the module $p$ modu-4-ps discovered in (Zhang et al., 2012a). Both positive-connection modules contain miRNA -146a, -150 and 223 and each is paired with a much smaller negative-connection module. More importantly, the functional enrichment profiles of the member genes in these two modules are similar. That is, the top over-represented gene ontology terms are consistently GO:0006955 immune response and the closely related ones. The involvement of miR-146a in the immune response has been widely investigated. Past studies suggest that miR-146a plays a functional role in immune response as a regulatory factor and its expression may serves as the 
passenger of disease and/or immune processes (Cameron et al., 2008; Lukiw et al., 2008; Hill et al., 2009; Curtale et al., 2010; Ji et al., 2010). This is exactly the main message conveyed by the module modu-1-ps (as well as the module p-modu-4-ps in (Zhang et al., 2012a)), where the positive correlations between the modular miRNAs and mRNAs could not be simply explained by a regulator-target mechanism. Another major finding from the modularity analysis is the predominant enrichment of immune response relevant genes in two semi-canonical regulatory modules (semi-CRMs) (i.e. modu-4-ne and modul-5-ne) and cell cycle process relevant genes in one semi-CRM (i.e. modu-2-ne). None of the modules derived from the prostate cancer samples (Zhang et al., 2012a) is comparable with them. This observation indicates that the regulatory roles of miRNAs in the immune response and cell cycle process are more widespread in ovarian carcinomas than in prostate tumors.

We proposed two algorithms to study the dynamicity of miRNA-mRNA interactions as cancer progresses. The first one is a regression model, which is similar to comparing the relationship between body weight and body height in the human population of different age phases. The model is statistically sound and biologically plausible. However, only $~ 400$ dynamic interactions with modest statistical significance were identified by this method. One major reason for the low efficiency is the relatively small sample sizes of the stage-IV and grade- 2 groups given the complexity of the regression model. In the second algorithm, the interaction level (strength) between a miRNA and an mRNA is regarded as a variable being evaluated for each individual sample rather than the sample group. This algorithm proved to be more efficient than the first one. Using the second method, we identified over 3000 dynamic miRNA-mRNA interactions. It is worth noting that, unlike the case in the comparison of PPC and MPC, our preliminary analysis showed that the somatic mutation-based partition of the TCGA ovarian carcinomas was not associated with the progression stages or serous grades. Therefore, in this regard, the identified dynamic interactions between the studied miRNAs and mRNAs should be mainly due to the dynamics in the cancer progression.

We summarize the dynamic miRNA-mRNA interactions and the implied biological insights as follows. First, the connection nodes are enriched with genes playing a role in cell cycle process and extracellular matrix (ECM). The former is related to a cancer hallmark and the latter is a dynamic niche in cancer progression (Lu et al., 2012). Second, the network constitution highlights the potential involvement of several miRNAs in carcinogenesis and cancer development. To the best of our knowledge, none of these involvements has been reported before. For example, miRNA-936 has not been documented as an onco-miRNA, tumor suppressor or progression biomarker. It was typically pinpointed in our analysis because of the association between its expression measurement and the survival time of ovarian cancer patients. As shown in Table S2, this miRNA has 613 connections with the mRNA genes, accounting for $18 \%$ of all the identified dynamic miRNA-mRNA interactions. Most of these relationships could be neglected if only a correlation analysis and family wise error rate (FWER) control procedure were performed on the un-stratified sample set. Third, the distribution profile of the identified miRNA-mRNA pairs indicates that a dynamic interaction tends to be present on the pairs outside the miRNA-mRNA modules. In other words, it suggests that miRNA-mRNA modules with strong correlations are relatively stable in cancer progression.

The prognostic application of gene expression signatures, especially in predicting the survival of cancer patients, has been the subject of many studies (Delfino and Rodriguez-Zas, 2013; Zhang et al., 2013). Emerging evidence indicates that more consistent signatures can be determined by 
the network-based approaches (Zhang et al., 2013). In this work, from the predictive genes preselected by the Cox-PH regression, we identified a prognostic signature with the member genes being included in the discovered modules. The predictive strength of this signature is robust on multiple patient cohorts as demonstrated by a classification method driven by the principal component (PC) analysis. In the implementation, the feature by which the patients were partitioned was the first or second PC score calculated from the expression matrix of the signature genes. It is well known that the leading PCs of a gene expression matrix represent the main co-expression patterns, which are usually related to some biological themes such as cancer subtypes (Alter et al., 2000; West et al., 2001; Wall M. E., 2003; Zhang et al., 2010). In this regard, the robustness of the identified prognostic signature over multiple independent datasets can be biologically explained. This observation also suggests the co-expression relationships between the mRNA genes as demonstrated in the miRNA-mRNA networks are largely conserved across different cohorts of high-grade serous ovarian carcinomas.

\section{Material and Methods}

TCGA datasets The gene expression profiling of 484 ovarian carcinomas (with recorded progression stages) was generated by TCGA. Among those samples, ten had no information regarding serous grades. The mRNA transcription levels of each tumor sample were measured on three different platforms, i.e. Affymetrix Human Exon1.0 ST Array, Agilent 244K Whole Genome Expression Array and Affymetrix HT-HG-U133A Array. Our analysis was based on the combined expression dataset of 11684 genes present on all three platforms (TCGA, 2011). The transcription levels of miRNAs were measured on Agilent-019118 Human miRNA Microarray 2.0. We downloaded the level-2 miRNA (normalized) expression data and the clinical data of the cancer patients from the TCGA database (http://cancergenome.nih.gov/). In the clinical dataset, cancer stages of patients were defined by the TNM system and the grades were defined by what the cells look like under a microscope (http://www.cancer.org/treatment/understandingyourdiagnosis/staging).

Validation cohorts We used five independent cohorts of ovarian cancer patients to validate the macro prognostic signature identified by Cox-PH regression and miRNA-mRNA network analysis. A brief profile of these cohorts is summarized in Table 2. The normalized expression data and clinical data were obtained from the Gene Expression Omnibus (GEO) repository (http://www.ncbi.nlm.nih.gov/geo/) and the ArrayExpress database (www.ebi.ac.uk/arrayexpress/). Before the survival analysis, we further processed these data by the following steps. First, if an individual sample was measured on multiple arrays (e.g. the Crijns dataset), the measurements were combined by calculating the median of the expression levels for each gene ID. Second, the expression values originally centered on the platformspecific gene IDs were adapted to the official gene symbols by calculating the mean for a gene with two or multiple gene IDs. Lastly, the adapted gene expression profiling of each cohort was double normalized using the quantile method.

miRNA set Eighty six "cancer-related" or/and "prognostic" miRNAs were focused in this study. The cancer-related batch contained 62 onco-miRNAs and tumor suppressor miRNAs as collected by (Wang et al., 2010). The prognostic batch contained 32 human miRNAs whose expression levels were associated with the overall survival time of the TCGA ovarian cancer patients ( $\mathrm{p}<$ 0.01). In identifying the prognostic miRNAs (and mRNA genes), we used a Cox-PH regression model with the gene expression level and the initial age of a patient as the independent variables. 
Condensed correlation network and identification of miRNA-mRNA Modules We employed an algorithm similar to the one used in our previous work (Zhang et al., 2012a). We organized the condensed correlation network (see Section 2) in the form of a matrix with mRNA genes as rows and miRNAs as columns. We filled in the matrix with 1 for the top positive miRNA-mRNA correlations, -1 for the top negative correlations, and 0 for the rest of the entries. Thus, a nonzero element represents a positive or negative correlation for a miRNA-mRNA pair. Using this matrix as the input, we generated a heatmap by applying the function "heatmap. 2 " in the $\mathrm{R}$ package "gplots". The layout of miRNAs and mRNAs in the heatmap was obtained by a twoway hierarchical clustering analysis with the Manhattan distance and Ward method as the arguments. We identified the miRNA-mRNA modules by the following three steps. (1) Based on the dendrogram and miRNA-mRNA connection patterns shown on the heatmap, five modular miRNA subsets (clusters) were visually determined. (2) For each of the miRNA subsets, the positive or negative connections with mRNAs were collected into a couple of two-column topology matrices, respectively; and (3) a miRNA-mRNA module pair was identified from the outputs of step (2) by removing the mRNAs with only one (positive or negative) connection.

Target site enrichment test Using a lab-owned R program (Zhang et al., 2012b) with the core being the matchPattern() function in the Bioconductor package "Biostrings" (http://www.bioconductor.org/packages/2.2/bioc/html/Biostrings.html), we identified the 7-mer and 8-mer miRNA target site motifs on the 3' UTR sequences (retrieved from hg-18) of the genes measured in the employed data. The binary miRNA-mRNA sequence affinity matrix (A) was then generated in a way such that an element $\left(A_{i j}\right)$ of value 1 indicated the existence of target site motif (s) for the $j^{\text {th }}$ miRNA in the $3^{\prime}$ UTR sequence of the $i^{\text {th }}$ mRNA. For a miRNA, the statistical significance of the target site enrichment level in the list of the correlated modular genes was measured by the Fisher's exact test in reference to the level of the entire gene set.

Survival analysis We performed the survival analysis using the statistical functions in the $\mathrm{R}$ package "survival" (Therneau, 2013). For the univariate survival analysis with a factorized variable as the predictor, the function "survdiff" was employed to generate the log-rank test pvalue. The Kaplan-Meier survival curves were obtained by the function "survfit". Multivariate survival analysis was conducted using the function "coxph" which implements the Cox Proportional Hazards regression (Therneau and Grambsch, 2000).

Regression model with heterogeneous slopes In this model, we assumed that there was a (cancer) progression-dependent regulatory relationship between a miRNA (regulator) and a mRNA gene whose 3'UTR contained at least one target site of the miRNA. The total expression level of other potentially regulatory miRNAs for the focused mRNA gene is included as a covariable in the regression model defined as follows.

$$
y_{j}=\mu+\alpha\left(x_{j}^{*}-x_{j}\right)+\sum_{k=1}^{m} I_{k j} \beta_{k}+\sum_{k=1}^{m} I_{k j} \gamma_{k} x_{j}+e_{j}
$$

In (1), $\mu, a, \beta_{k}$ and $\gamma_{k}$ are the model parameters (the intercept or regression coefficients), and $\boldsymbol{e}_{j}$ denotes the noise. $\mathbf{y}=\left(y_{1}, y_{2} \ldots \ldots y_{q}\right)$ and $\mathbf{x}=\left(x_{1}, x_{2} \ldots \ldots x_{q}\right)$ are the mRNA and miRNA expression vectors of $q$ cancer samples, respectively. $\mathbf{x}^{*}=\left(x_{1}^{*}, x_{2}^{*} \ldots \ldots x_{q}^{*}\right)$ is the vector for the total expression quantities of all miRNAs with each having at least one 7-mer or 8-mer miRNA target site on the 3'UTR of the focused mRNA gene. $\mathbf{I}_{k}=\left(I_{k 1}, I_{k 2} \ldots \ldots I_{k q}\right)$ represents the 
indicator vector (with values 0 or 1 ) for the $\mathrm{k}^{\text {th }}$ group $(1 \leq \mathrm{k} \leq \mathrm{m})$, assuming that the $q$ cancer samples are partitioned into $m$ clinical groups. In the dynamicity analysis, the clinical groups were established with respect to cancer progression stages $(m=3)$ or serous grades $(m=2)$. For the stage-group focused modelling, the $\mathrm{p}$-values of interest were calculated via three t-tests with $H_{0}: \gamma_{1}-\gamma_{2}=0, H_{0}: \gamma_{1}-\gamma_{3}=0$ or $H_{0}: \gamma_{2}-\gamma_{3}=0$ as the null hypothesis, respectively. For the grade-group focused modelling, the p-value was obtained by a t-test with $H_{0}: \gamma_{1}-\gamma_{2}=0$ as the null hypothesis. We performed the linear model analysis using the statistical function $l m$ included in the R package "stat".

Interaction strength (IS) We proposed a novel algorithm to measure the strength of samplespecific miRNA-mRNA interaction (Hecker et al., 2013). It includes the following steps. First, using a rank-based method which replaces each entry in one array by its position (rank) in the ordered array counted from the smallest value divided by the total number of genes (Qiu et al., 2013), we double normalized the mRNA gene (miRNA) expression levels of each cancer sample in the TCGA cohort. The normalized values were uniformly distributed in $[0,1]$ and called expression ranks (ERs). The baseline ER of each mRNA gene (or miRNA) was then estimated by the average of its ER values in the two baseline samples (see Section 2 for details).

Accordingly, the rank transition of an mRNA gene (miRNA) present in each non-baseline sample was calculated by subtracting the baseline ER from its ER. Lastly, the sample-specific interaction strength (IS) for a pair of miRNA and mRNA was obtained by the product of the corresponding rank transitions.

Estimation of FDR We adapted the permutation-based method in (Pickrell et al., 2010) to estimate the false discovery rate (FDR) in studying the dynamicity of miRNA-mRNA interactions. For each miRNA-mRNA pair, we permuted the indicators of the clinical groups five times, re-conducted the regression (and non-parametric) analysis, and recorded the p-values for the statistics of interest. By doing so, we established the model-specific empirical null distributions for the $\mathrm{p}$-values. We then compared a true distribution of $\mathrm{p}$-values to the corresponding null distribution to estimate FDR. That is, we found a p-value threshold $\mathrm{z}$ such that $P(p 0<z) / P(p 1<z)=x$, where $x$ is the desired FDR, $p 0$ is a $\mathrm{p}$-value from the null distribution, $p 1$ is a $\mathrm{p}$-value from the true distribution, $P(p O<z)$ is the fraction of $\mathrm{p}$-values from the permutations that fall below the threshold, and similarly, $P(p 1<z)$ is the corresponding fraction in the non-permuted data.

\section{Acknowledgements}

This publication was made possible by funding from the NIH NIMHD-RCMI grant \#2G12MD007595, the DOD ARO grant \# W911NF-15-1-0510 and the Louisiana Cancer Research Consortium (LCRC). The contents are solely the responsibility of the authors and do not necessarily represent the official views of the NIH, DOD or LCRC.

\section{References}

TCGA, 2011. Integrated genomic analyses of ovarian carcinoma. Nature 474, 609-615. Aguda, B.D., Kim, Y., Piper-Hunter, M.G., Friedman, A., Marsh, C.B., 2008. MicroRNA regulation of a cancer network: consequences of the feedback loops involving miR-17-92, E2F, and Myc. Proc Natl Acad Sci U S A 105, 19678-19683. 
Alter, O., Brown, P.O., Botstein, D., 2000. Singular value decomposition for genome-wide expression data processing and modeling. Proc Natl Acad Sci U S A 97, 10101-10106.

Bandyopadhyay, S., Mitra, R., Maulik, U., Zhang, M.Q., 2010. Development of the human cancer microRNA network. Silence 1, 6 .

Barabasi, A.L., Gulbahce, N., Loscalzo, J., 2011. Network medicine: a network-based approach to human disease. Nat Rev Genet 12, 56-68.

Bartel, D.P., 2009. MicroRNAs: target recognition and regulatory functions. Cell 136, 215-233.

Bast, R.C., Jr., Hennessy, B., Mills, G.B., 2009. The biology of ovarian cancer: new opportunities for translation. Nat Rev Cancer 9, 415-428.

Bentink, S., Haibe-Kains, B., Risch, T., Fan, J.B., Hirsch, M.S., Holton, K., Rubio, R., April, C., Chen, J., Wickham-Garcia, E., Liu, J., Culhane, A., Drapkin, R., Quackenbush, J., Matulonis, U.A., 2012.

Angiogenic mRNA and microRNA gene expression signature predicts a novel subtype of serous ovarian cancer. PLoS One 7, e30269.

Bisognin, A., Sales, G., Coppe, A., Bortoluzzi, S., Romualdi, C., 2012. MAGIA(2): from miRNA and genes expression data integrative analysis to microRNA-transcription factor mixed regulatory circuits (2012 update). Nucleic Acids Res 40, W13-21.

Bonome, T., Levine, D.A., Shih, J., Randonovich, M., Pise-Masison, C.A., Bogomolniy, F., Ozbun, L., Brady, J., Barrett, J.C., Boyd, J., Birrer, M.J., 2008. A gene signature predicting for survival in suboptimally debulked patients with ovarian cancer. Cancer Res 68, 5478-5486.

Cameron, J.E., Yin, Q., Fewell, C., Lacey, M., McBride, J., Wang, X., Lin, Z., Schaefer, B.C., Flemington, E.K., 2008. Epstein-Barr virus latent membrane protein 1 induces cellular MicroRNA miR-146a, a modulator of lymphocyte signaling pathways. J Virol 82, 1946-1958.

Cheng, Y., Kim, J.W., Liu, W., Dunn, T.A., Luo, J., Loza, M.J., Kim, S.T., Zheng, S.L., Xu, J., Isaacs, W.B., Chang, B.L., 2009. Genetic and epigenetic inactivation of TNFRSF10C in human prostate cancer.

Prostate 69, 327-335.

Crijns, A.P., Fehrmann, R.S., de Jong, S., Gerbens, F., Meersma, G.J., Klip, H.G., Hollema, H., Hofstra, R.M., te Meerman, G.J., de Vries, E.G., van der Zee, A.G., 2009. Survival-related profile, pathways, and transcription factors in ovarian cancer. PLoS Med 6, e24.

Curtale, G., Citarella, F., Carissimi, C., Goldoni, M., Carucci, N., Fulci, V., Franceschini, D., Meloni, F., Barnaba, V., Macino, G., 2010. An emerging player in the adaptive immune response: microRNA-146a is a modulator of IL-2 expression and activation-induced cell death in T lymphocytes. Blood 115, 265-

273.

Delfino, K.R., Rodriguez-Zas, S.L., 2013. Transcription factor-microRNA-target gene networks associated with ovarian cancer survival and recurrence. PLoS One 8, e58608.

Dvinge, H., Git, A., Graf, S., Salmon-Divon, M., Curtis, C., Sottoriva, A., Zhao, Y., Hirst, M., Armisen, J., Miska, E.A., Chin, S.F., Provenzano, E., Turashvili, G., Green, A., Ellis, I., Aparicio, S., Caldas, C., 2013. The shaping and functional consequences of the microRNA landscape in breast cancer. Nature 497, 378-382.

Filipowicz, W., Bhattacharyya, S.N., Sonenberg, N., 2008. Mechanisms of post-transcriptional regulation by microRNAs: are the answers in sight? Nat Rev Genet 9, 102-114.

Hanahan, D., Weinberg, R.A., 2011. Hallmarks of cancer: the next generation. Cell 144, 646-674. Hecker, N., Stephan, C., Mollenkopf, H.J., Jung, K., Preissner, R., Meyer, H.A., 2013. A new algorithm for integrated analysis of miRNA-mRNA interactions based on individual classification reveals insights into bladder cancer. PLoS One 8, e64543.

Hill, J.M., Zhao, Y., Clement, C., Neumann, D.M., Lukiw, W.J., 2009. HSV-1 infection of human brain cells induces miRNA-146a and Alzheimer-type inflammatory signaling. Neuroreport 20, 1500-1505. 
Huang da, W., Sherman, B.T., Tan, Q., Collins, J.R., Alvord, W.G., Roayaei, J., Stephens, R., Baseler, M.W., Lane, H.C., Lempicki, R.A., 2007. The DAVID Gene Functional Classification Tool: a novel biological module-centric algorithm to functionally analyze large gene lists. Genome Biol 8, R183. Jayaswal, V., Lutherborrow, M., Ma, D.D., Yang, Y.H., 2011. Identification of microRNA-mRNA modules using microarray data. BMC Genomics 12, 138.

Ji, X., Tang, J., Halberg, R., Busam, D., Ferriera, S., Pena, M.M., Venkataramu, C., Yeatman, T.J., Zhao, S., 2010. Distinguishing between cancer driver and passenger gene alteration candidates via crossspecies comparison: a pilot study. BMC Cancer 10, 426.

Joung, J.G., Hwang, K.B., Nam, J.W., Kim, S.J., Zhang, B.T., 2007. Discovery of microRNA-mRNA modules via population-based probabilistic learning. Bioinformatics 23, 1141-1147.

Li, J., Fadare, O., Xiang, L., Kong, B., Zheng, W., 2012. Ovarian serous carcinoma: recent concepts on its origin and carcinogenesis. J Hematol Oncol 5, 8.

Lu, P., Weaver, V.M., Werb, Z., 2012. The extracellular matrix: a dynamic niche in cancer progression. J Cell Biol 196, 395-406.

Lukiw, W.J., Zhao, Y., Cui, J.G., 2008. An NF-kappaB-sensitive micro RNA-146a-mediated inflammatory circuit in Alzheimer disease and in stressed human brain cells. J Biol Chem 283, 31315-31322.

Michoel, T., De Smet, R., Joshi, A., Van de Peer, Y., Marchal, K., 2009. Comparative analysis of modulebased versus direct methods for reverse-engineering transcriptional regulatory networks. BMC Syst Biol 3, 49.

Newman, M.E., 2006. Modularity and community structure in networks. Proc Natl Acad Sci U S A 103, 8577-8582.

O'Day, E., Lal, A., 2010. MicroRNAs and their target gene networks in breast cancer. Breast Cancer Res 12, 201.

Peng, X., Guo, W., Liu, T., Wang, X., Tu, X., Xiong, D., Chen, S., Lai, Y., Du, H., Chen, G., Liu, G., Tang, Y., Huang, S., Zou, X., 2011. Identification of miRs-143 and -145 that is associated with bone metastasis of prostate cancer and involved in the regulation of EMT. PLoS One 6, e20341.

Peng, X., Li, Y., Walters, K.A., Rosenzweig, E.R., Lederer, S.L., Aicher, L.D., Proll, S., Katze, M.G., 2009. Computational identification of hepatitis $C$ virus associated microRNA-mRNA regulatory modules in human livers. BMC Genomics 10, 373.

Pickrell, J.K., Marioni, J.C., Pai, A.A., Degner, J.F., Engelhardt, B.E., Nkadori, E., Veyrieras, J.B., Stephens, M., Gilad, Y., Pritchard, J.K., 2010. Understanding mechanisms underlying human gene expression variation with RNA sequencing. Nature 464, 768-772.

Qiu, X., Wu, H., Hu, R., 2013. The impact of quantile and rank normalization procedures on the testing power of gene differential expression analysis. BMC Bioinformatics 14, 124.

Saito, Y., Kojima, T., Takahashi, N., 2012. Mab21/2 is essential for embryonic heart and liver development. PLoS One 7, e32991.

Schwarz, A.J., Gozzi, A., Bifone, A., 2008. Community structure and modularity in networks of correlated brain activity. Magn Reson Imaging 26, 914-920.

Siegel, R., Ma, J., Zou, Z., Jemal, A., 2014. Cancer statistics, 2014. CA Cancer J Clin 64, 9-29.

Sumazin, P., Yang, X., Chiu, H.S., Chung, W.J., lyer, A., Llobet-Navas, D., Rajbhandari, P., Bansal, M., Guarnieri, P., Silva, J., Califano, A., 2011. An extensive microRNA-mediated network of RNA-RNA interactions regulates established oncogenic pathways in glioblastoma. Cell 147, 370-381. Therneau, T., 2013. A Package for Survival Analysis in S. R package version 2.37-4. http://CRAN.Rproject.org/package=survival. .

Therneau, T.M., Grambsch, P.M., 2000. Modeling survival data : extending the Cox model. Springer, New York.

Tothill, R.W., Tinker, A.V., George, J., Brown, R., Fox, S.B., Lade, S., Johnson, D.S., Trivett, M.K., Etemadmoghadam, D., Locandro, B., Traficante, N., Fereday, S., Hung, J.A., Chiew, Y.E., Haviv, I., 
Gertig, D., DeFazio, A., Bowtell, D.D., 2008. Novel molecular subtypes of serous and endometrioid ovarian cancer linked to clinical outcome. Clin Cancer Res 14, 5198-5208.

Tran, D.H., Satou, K., Ho, T.B., 2008. Finding microRNA regulatory modules in human genome using rule induction. BMC Bioinformatics 9 Suppl 12, S5.

Visone, R., Croce, C.M., 2009. MiRNAs and cancer. Am J Pathol 174, 1131-1138.

Wall M. E., R.A., Rocha L. A., 2003. Singular value decomposition and principal component analysis. In:

Berrar D. P, D.W., Granzow M. (Ed.), A Practical Approach to Microarray Data Analysis. Kluwer

Norwell, MA, Norwell, MA, pp. 91-109.

Wang, D., Qiu, C., Zhang, H., Wang, J., Cui, Q., Yin, Y., 2010. Human microRNA oncogenes and tumor suppressors show significantly different biological patterns: from functions to targets. PLoS One 5. West, M., Blanchette, C., Dressman, H., Huang, E., Ishida, S., Spang, R., Zuzan, H., Olson, J.A., Jr., Marks, J.R., Nevins, J.R., 2001. Predicting the clinical status of human breast cancer by using gene expression profiles. Proc Natl Acad Sci U S A 98, 11462-11467.

Yin, Y., Yan, Z.P., Lu, N.N., Xu, Q., He, J., Qian, X., Yu, J., Guan, X., Jiang, B.H., Liu, L.Z., 2013.

Downregulation of miR-145 associated with cancer progression and VEGF transcriptional activation by targeting N-RAS and IRS1. Biochim Biophys Acta 1829, 239-247.

Yoshihara, K., Tsunoda, T., Shigemizu, D., Fujiwara, H., Hatae, M., Masuzaki, H., Katabuchi, H., Kawakami, Y., Okamoto, A., Nogawa, T., Matsumura, N., Udagawa, Y., Saito, T., Itamochi, H., Takano, M., Miyagi, E., Sudo, T., Ushijima, K., Iwase, H., Seki, H., Terao, Y., Enomoto, T., Mikami, M., Akazawa, K., Tsuda, H., Moriya, T., Tajima, A., Inoue, I., Tanaka, K., 2012. High-risk ovarian cancer based on 126gene expression signature is uniquely characterized by downregulation of antigen presentation pathway. Clin Cancer Res 18, 1374-1385.

Zhang, W., Edwards, A., Fan, W., Flemington, E.K., Zhang, K., 2012a. miRNA-mRNA correlationnetwork modules in human prostate cancer and the differences between primary and metastatic tumor subtypes. PLoS One 7, e40130.

Zhang, W., Edwards, A., Fan, W., Zhu, D., Zhang, K., 2010. svdPPCS: an effective singular value decomposition-based method for conserved and divergent co-expression gene module identification. BMC Bioinformatics 11, 338.

Zhang, W., Edwards, A., Zhu, D., Flemington, E.K., Deininger, P., Zhang, K., 2012b. miRNA-mediated relationships between Cis-SNP genotypes and transcript intensities in lymphocyte cell lines. PLoS One 7, e31429.

Zhang, W., Ota, T., Shridhar, V., Chien, J., Wu, B., Kuang, R., 2013. Network-based survival analysis reveals subnetwork signatures for predicting outcomes of ovarian cancer treatment. PLoS Comput Biol 9, e1002975. 


\section{Figure captions}

Fig. 1. The scheme of study flow.

Fig. 2. Identifying miRNA-mRNA correlation-network modules by a hierarchical clustering algorithm. Red: the top positive correlations. Yellow: the top negative correlations. Orange: pseudo or unconsidered correlations.

Fig. 3. The miRNA target site enrichment analysis for four major negative connection miRNA-mRNA modules.

Fig. 4. Illustrating the (cancer) progression-stage relevant regression of the expression level of an mRNA gene (e.g. FOXF1) on the expression level of a miRNA (e.g. miR-17 or let-7g).

Fig. 5. An illustration of miRNA-mRNA interaction strengths over different (cancer) progression stages. $\mathbf{X}$-axis: cancer progression stages. Y-axis: miRNA-mRNA interaction strengths (IS) of a specific mRNA-miRNA pair. All the mRNA genes presented in the 14 plots have been annotated to the KEGG pathway $\sim$ ECM-receptor-interaction. See the narrative for statistical inference about IS. Box color: "purple (yellow)" indicates that the miRNA expression level of the cancer samples in the group is significantly up- (down-) regulated ( $p<0.01)$ compared to the baseline - the combination of two stage-IIA samples; "white" represents that the miRNA expression level of the cancer samples in the group is not significantly different ( $\mathrm{p}>$ $0.01)$ from the baseline. The statistical inference regarding the regulation of a miRNA was performed by first converting the rank transitions (RT, see Method section) into a vector with values 1 or -1 according to the sign of the original value, and then testing the null hypothesis that the transformed RT measurements follow the Binomial(n, 0.5), where $\mathrm{n}$ is the size of the corresponding tumor group.

Fig. 6. The comparison of modular and dynamic miRNA-mRNA interactions. A: S1 (modular interaction) and S2 (dynamic interaction) are two subsets of a larger relationship set (S0), which contains $\sim 271 \mathrm{~K}$ top miRNA-mRNA connections (interactions). B (C): the distribution profile of the network connections in S1 (S2) regarding the involved miRNAs. The frequency (Y-axis) represents the number of mRNA genes associated with the corresponding miRNA.

Fig. 7. The performance of the selected prognostic signatures. PC1(PC2): the first (second) principal component score of a gene expression matrix. For example, TCGA-modu-PC2 (Bonome-modu-PC2): shows the result of survival analysis for the TCGA ( $\underline{\text { Bonome }})$ cohort when the PC2 of the "modu" gene expression matrix is used as the classification feature in the log-rank rest and a predictive variable in the Cox-PH regression analysis. "modu" denotes that the genes in the subset G1. (See Section 2.4 for details) 


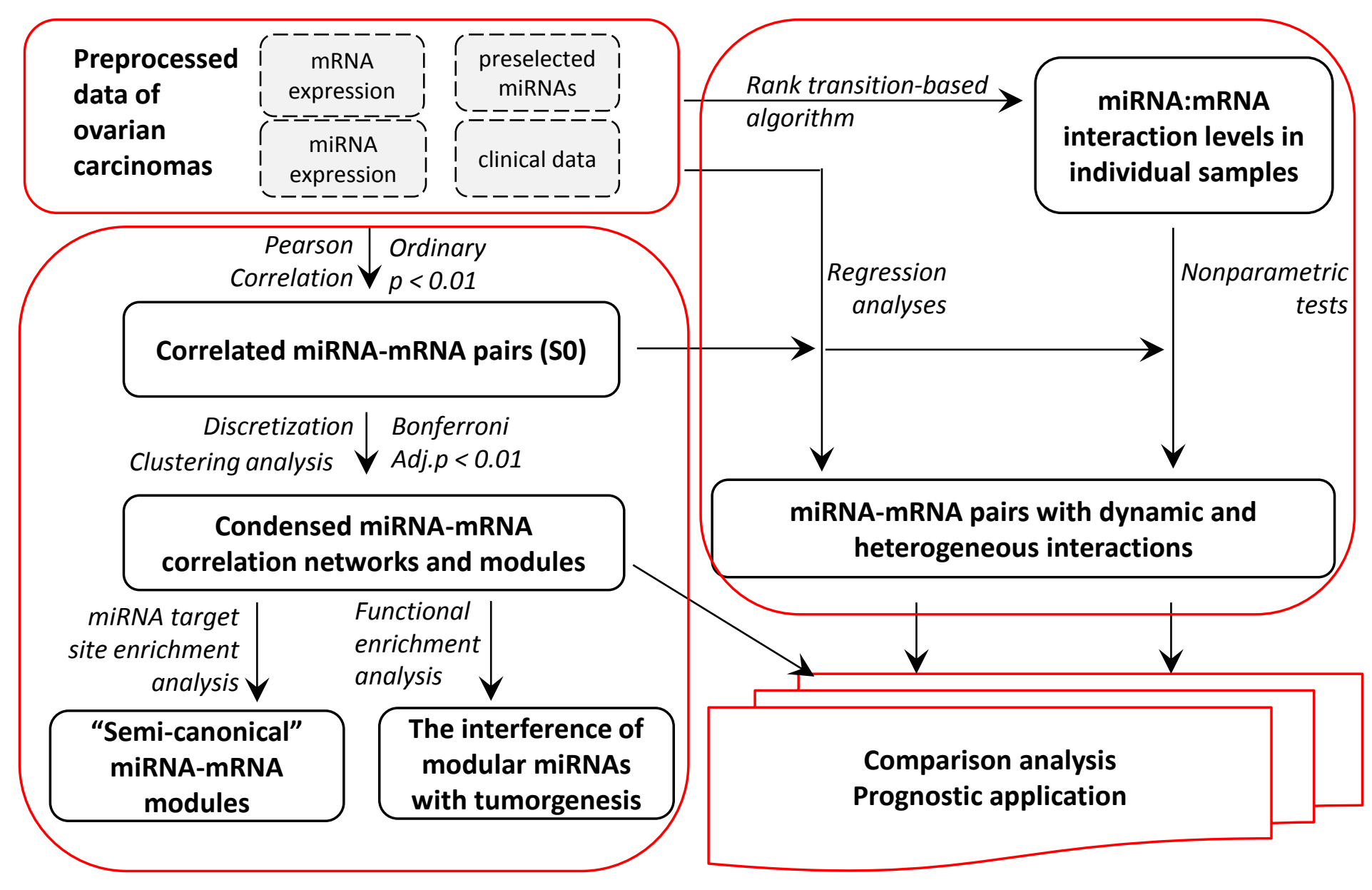




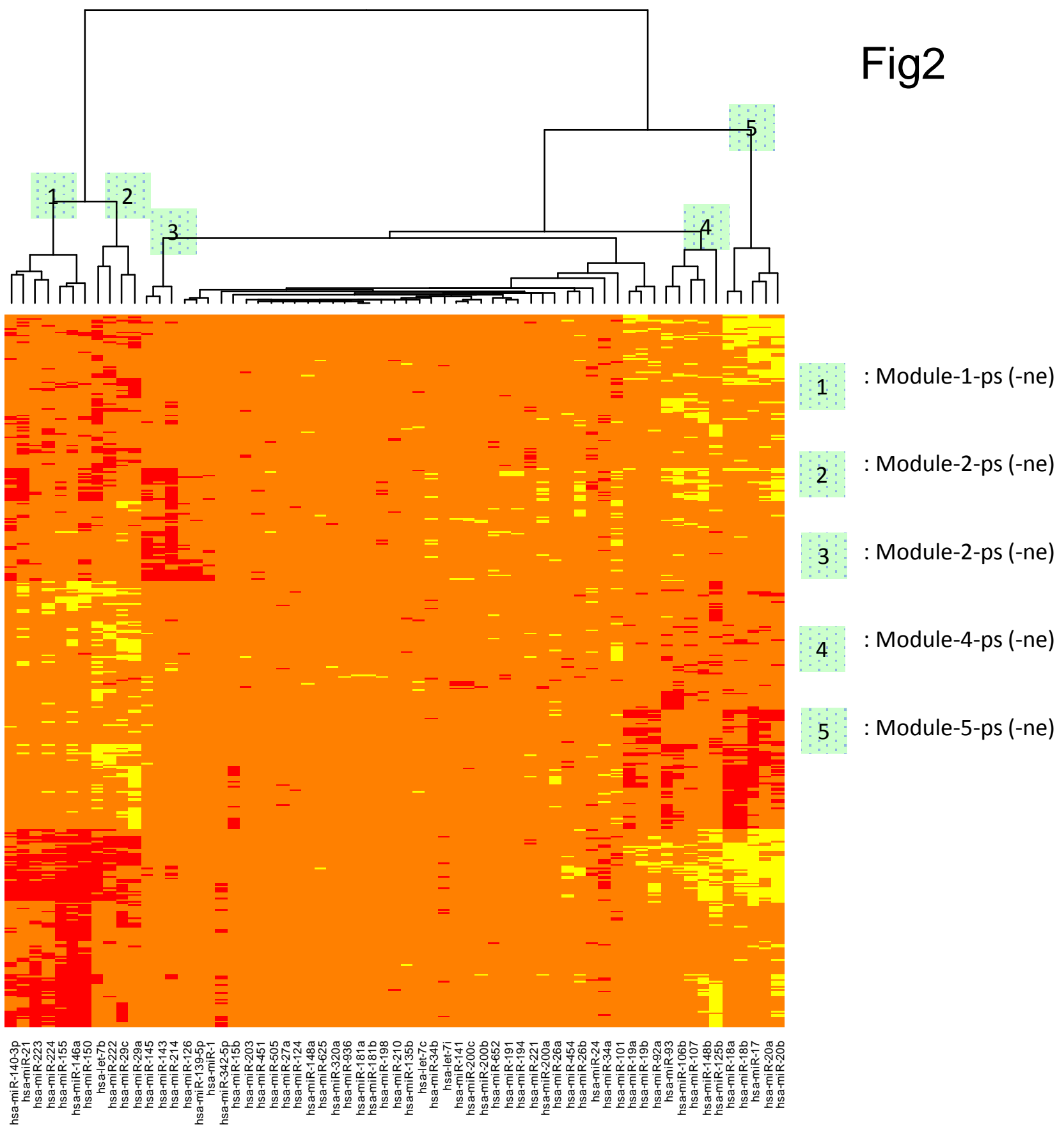


stage III

$p$-value $=4.75 \mathrm{e}-01$

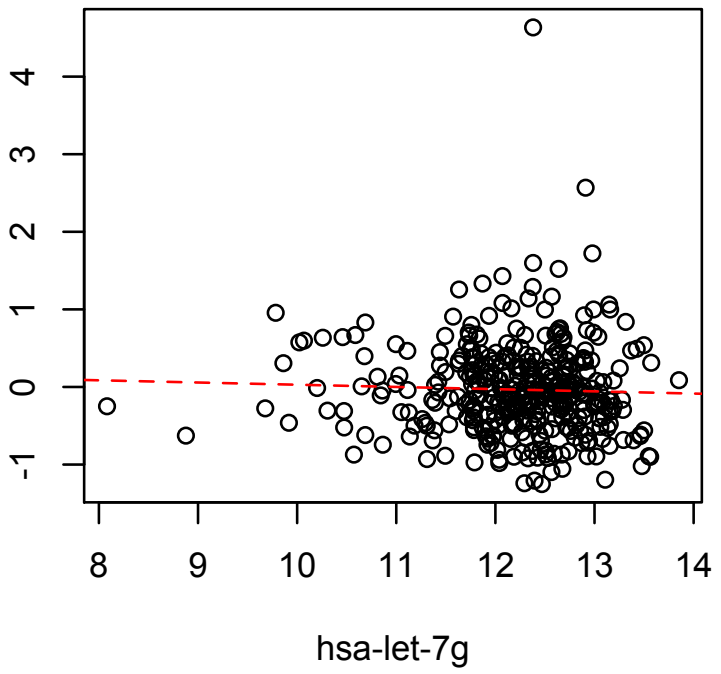

stage III

$p$-value $=2.388 \mathrm{e}-01$

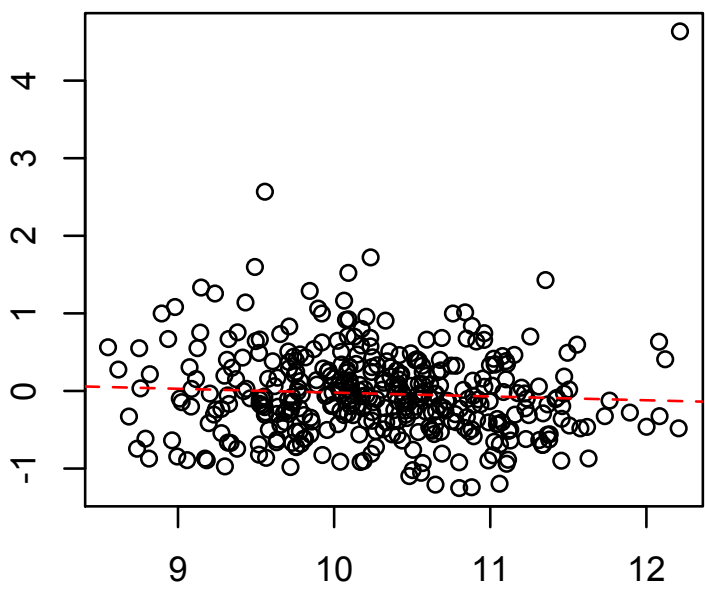

hsa-miR-17 stage IV fig4

$p$-value $=1.944 \mathrm{e}-07$

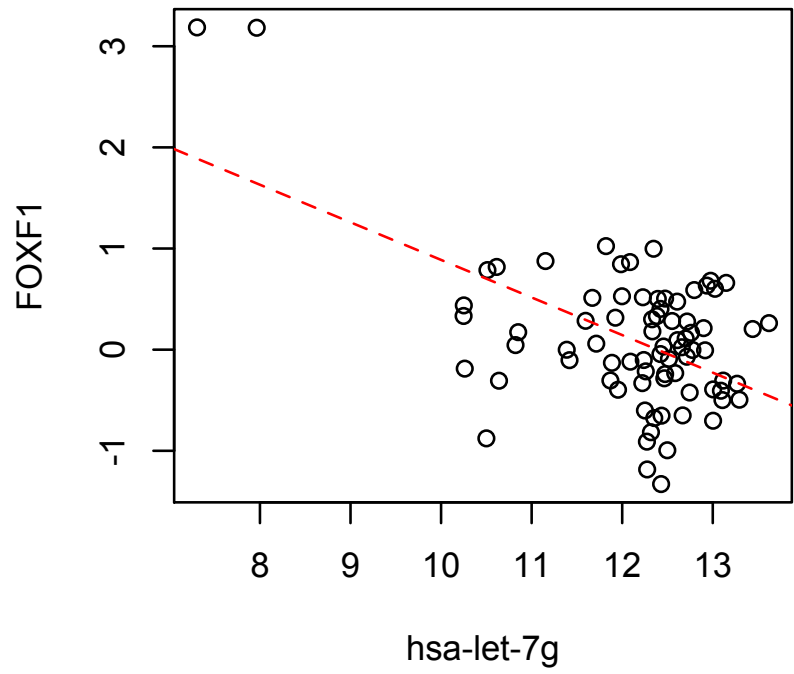

stage IV

$p$-value $=2.578 \mathrm{e}-06$

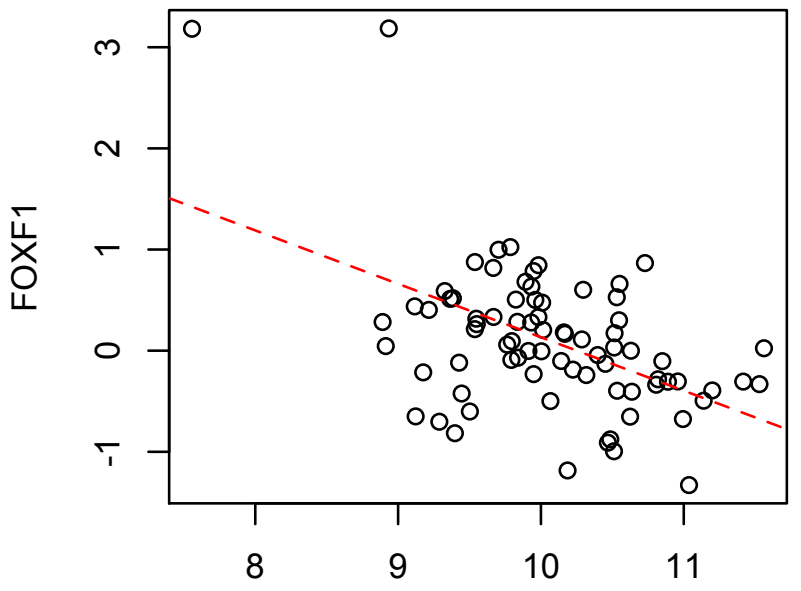

hsa-miR-17 


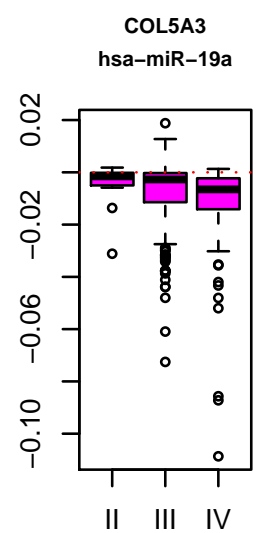

COL5A1

hsa-miR-19a

THBS2

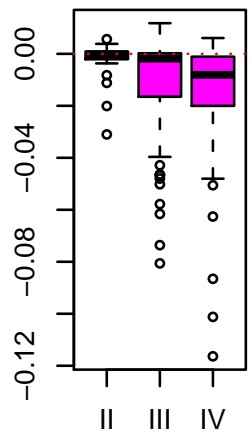

THBS4

hsa-miR-145

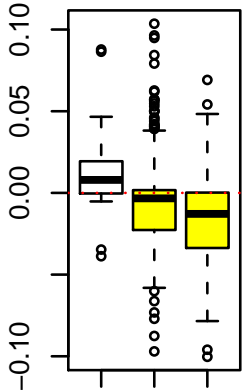

II III IV

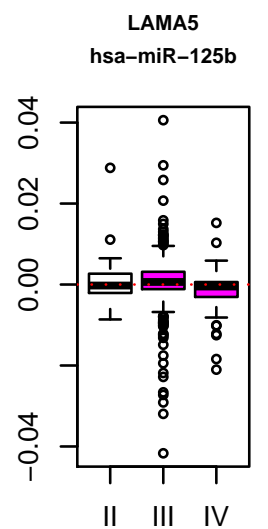

COL5A3

hsa-miR-145

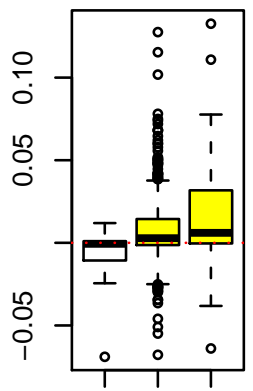

II III IV

COL2A1

hsa-miR-19a

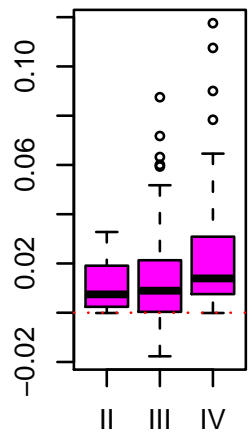

hsa-miR-19a

hsa-miR-19a

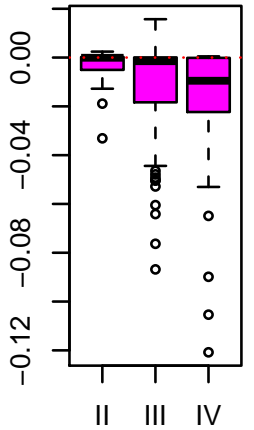

THBS1

hsa-miR-145

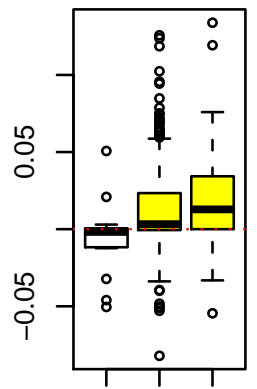

II III IV

hsa-miR-145

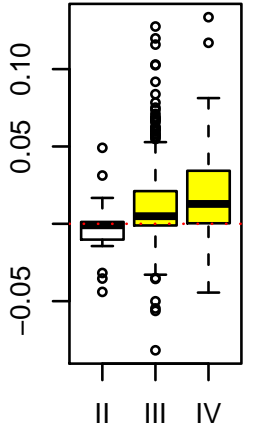

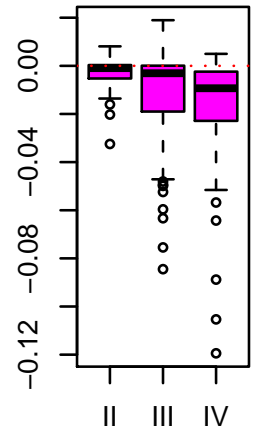

COL5A2

hsa-miR-145

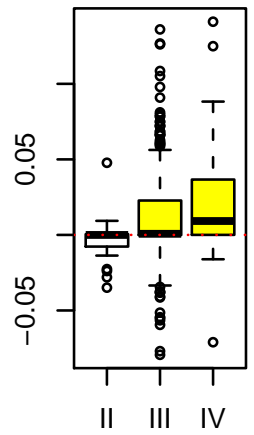

COL3A1

hsa-miR-145

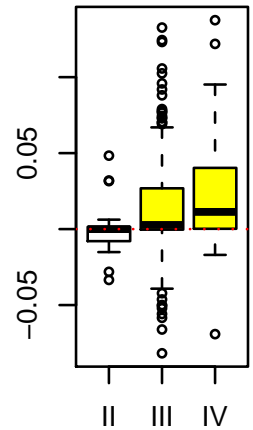

HMMR

hsa-miR-224

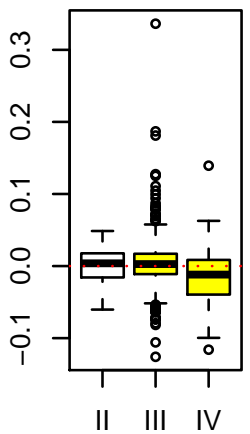

II III IV

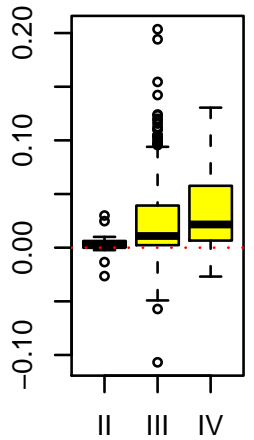




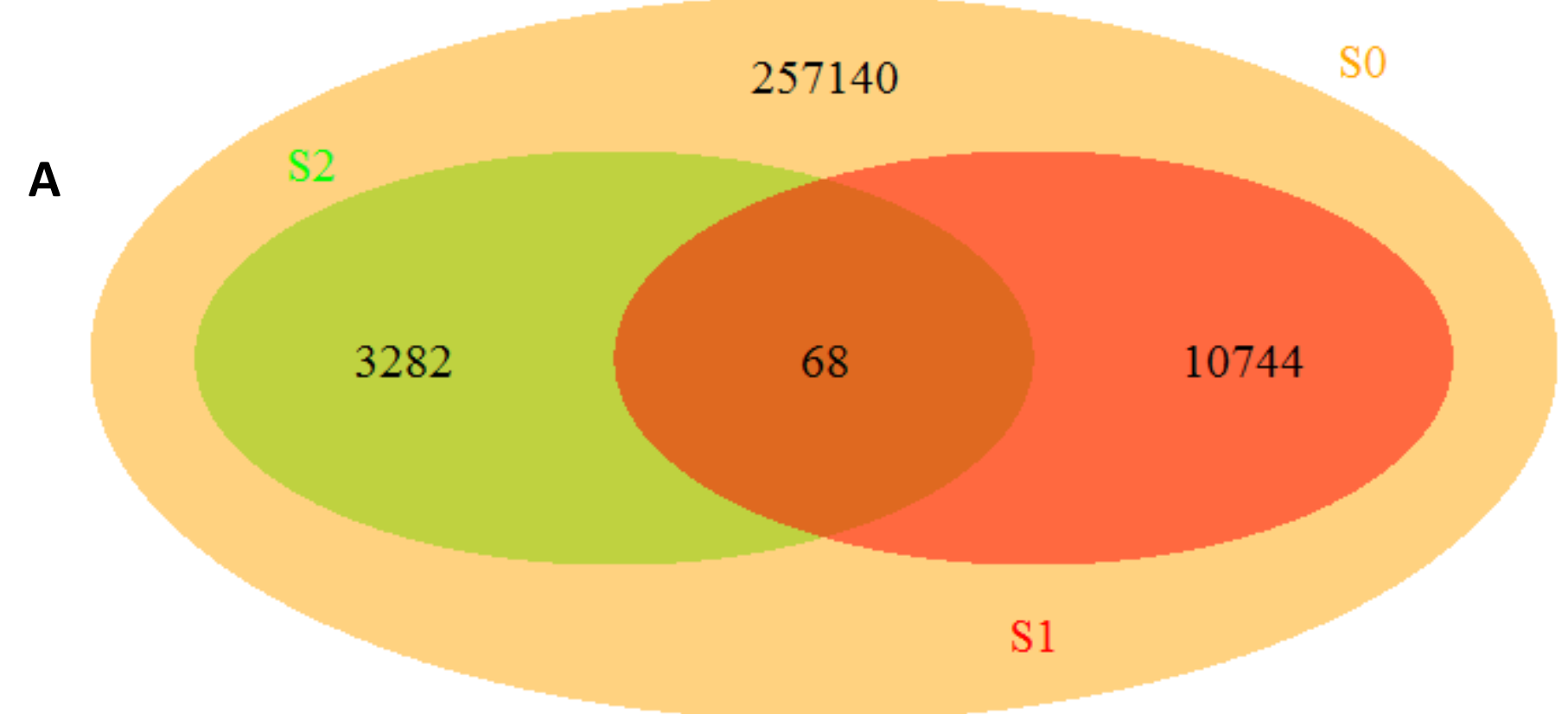

Fig6

B
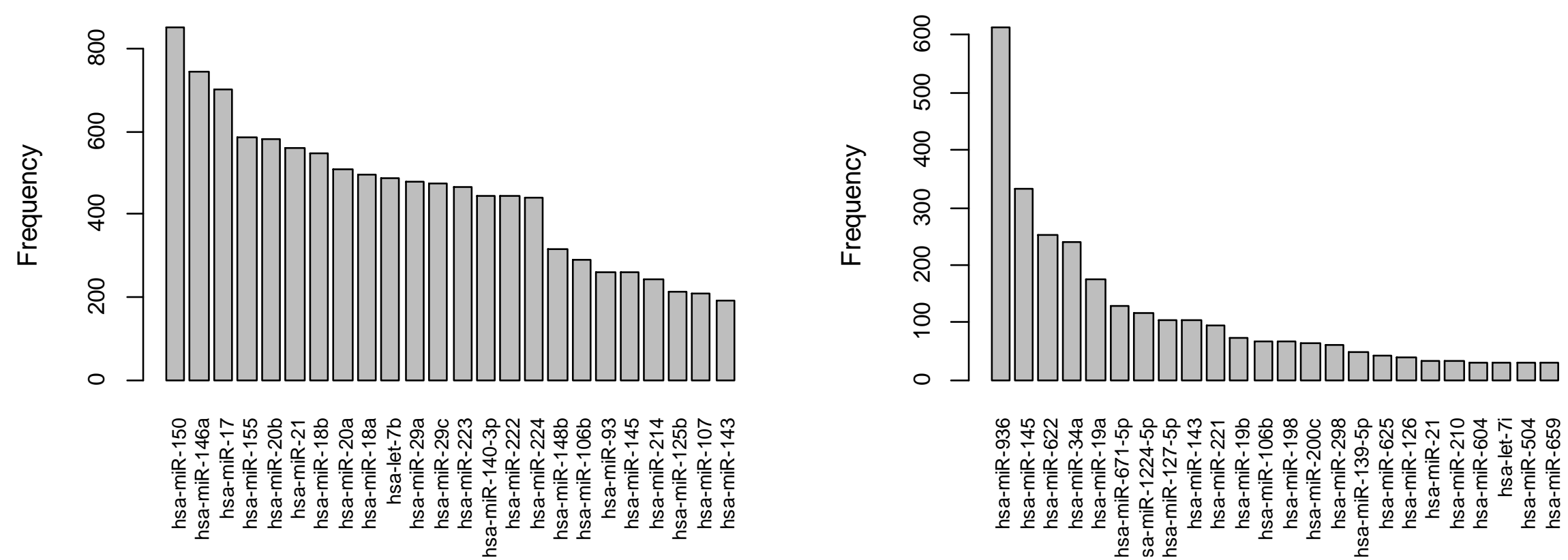
Logrank test p.value: $5.01 \mathrm{e}-10$

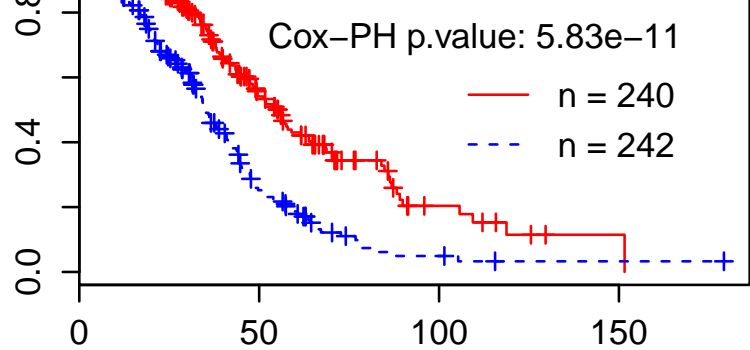

Overall survival months

\section{Bonome-modu-PC2}

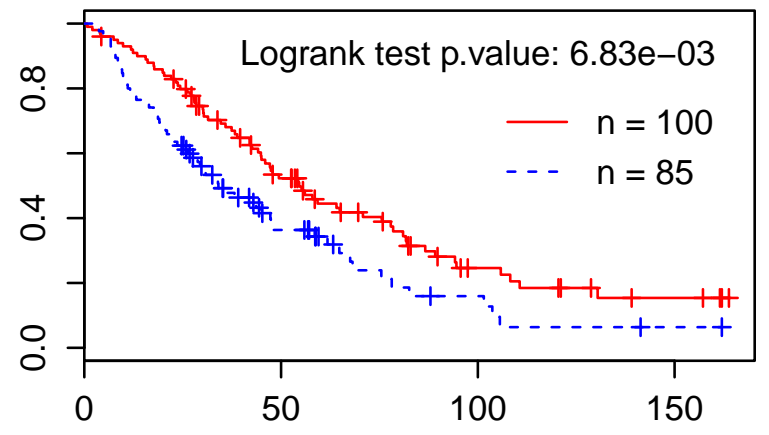

Overall survival months

\section{Tothill-mudu-PC2}

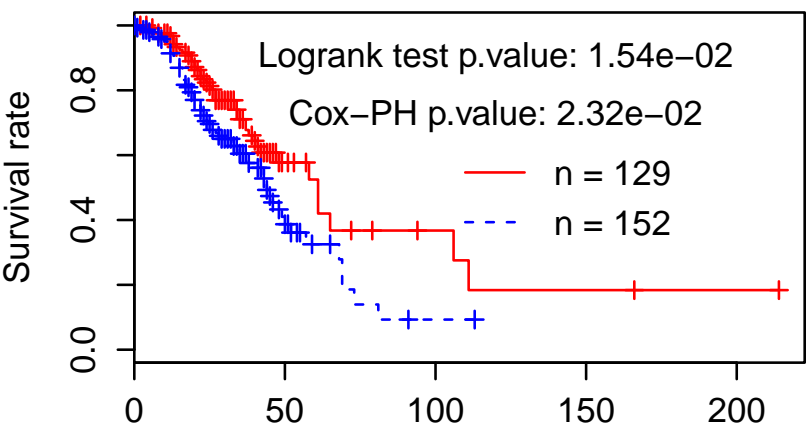

Overall survival months

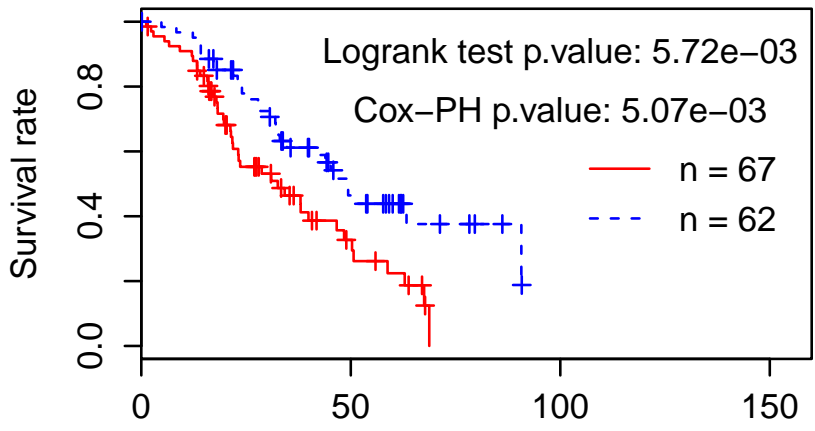

Overall survival months

\section{Crijns-modu-PC1}

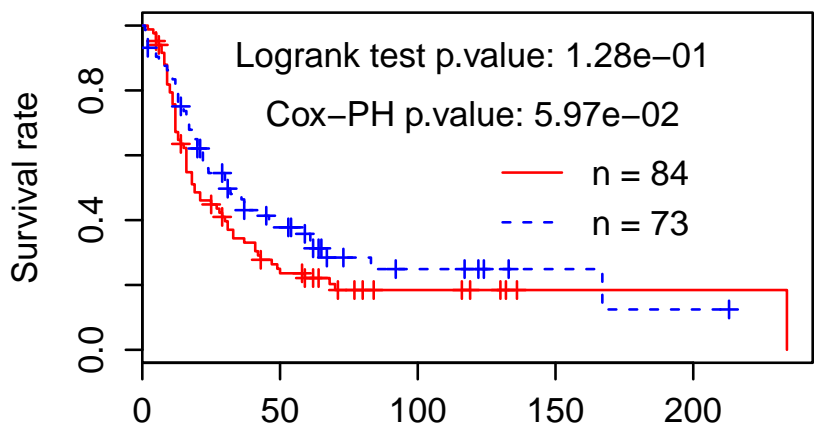

Overall survival months

\section{Yoshihara-modu-PC1}

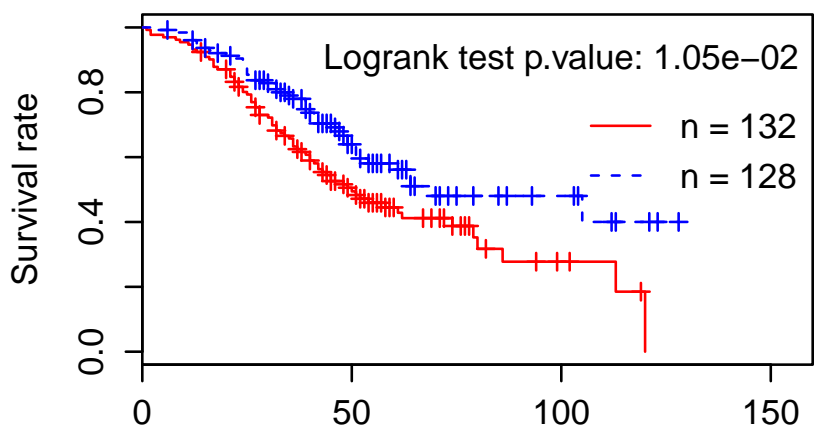

Overall survival months 
Table 1 The profile of miRNA-mRNA correlation modules

\begin{tabular}{|c|c|c|c|}
\hline Module IDs & \# mRNA genes & miRNAs & Top over-represented GO terms \\
\hline modu-1-ne & 139 & $\begin{array}{l}\text { miRNA-146a, - } \\
150,-155,-21,- \\
223,-224\end{array}$ & $\begin{array}{l}\text { GO:0031012 extracellular matrix }\left(1.57 \mathrm{e}-03^{\mathrm{b}}\right) \\
\text { GO:0005578 proteinaceous extracellular matrix }(1.85 \mathrm{e}-03) \\
\text { GO:0044421 extracellular region part(5.19e-03) } \\
\text { GO:0008270 zinc ion binding(3.84e-02) } \\
\text { GO:0048598 embryonic morphogenesis(3.82e-01) }\end{array}$ \\
\hline modu-1-ps & 873 & $\begin{array}{l}\text { miRNA-140-3p, - } \\
\text { 146a, -150, -155, - } \\
21,-223,-224\end{array}$ & $\begin{array}{l}\text { GO:0006955 immune response(6.66e-100) } \\
\text { GO:0006952 defense response( } 5.62 \mathrm{e}-67) \\
\text { GO:0006954 inflammatory response( } 1.66 \mathrm{e}-40) \\
\text { GO:0009611 response to wounding( } 3.53 \mathrm{e}-36) \\
\text { GO:0002684 positive regulation of immune system process( } 3.86 \mathrm{e}-36)\end{array}$ \\
\hline modu-2-ne ${ }^{a}$ & 282 & $\begin{array}{l}\text { hsa-let-7b; } \\
\text { miRNA-222, -29a, } \\
-29 c\end{array}$ & $\begin{array}{l}\text { GO:0022403 cell cycle phase(1.37e-11) } \\
\text { GO:0022402 cell cycle process(5.08e-11) } \\
\text { GO:0000278 mitotic cell cycle(3.97e-11) } \\
\text { GO:0000279 M phase(7.92e-11) } \\
\text { GO:0007049 cell cycle(9.00e-11) }\end{array}$ \\
\hline modu-2-ps & 447 & $\begin{array}{l}\text { hsa-let-7b; } \\
\text { miRNA-222, -29a, } \\
-29 c\end{array}$ & $\begin{array}{l}\text { GO:0006955 immune response(1.63e-38) } \\
\text { GO:0006952 defense response(5.07e-26) } \\
\text { GO:0006954 inflammatory response(6.79e-15) } \\
\text { GO:0009611 response to wounding(5.14e-15) } \\
\text { GO:0002684 positive regulation of immune system process(5.12e-15) }\end{array}$ \\
\hline modu-3-ps & 270 & $\begin{array}{l}\text { miRNA-143, }-145 \\
-214\end{array}$ & $\begin{array}{l}\text { GO:0005576 extracellular region(2.90e-35) } \\
\text { GO:0044421 extracellular region part(1.95e-34) GO:0031012 extracellular } \\
\text { matrix(2.80e-30) } \\
\text { GO:0022610 biological adhesion(6.80e-28) } \\
\text { GO:0007155 cell adhesion(6.80e-28) }\end{array}$ \\
\hline modu-3-ne & 2 & $\begin{array}{l}\text { miRNA-143, }-145 \\
-214\end{array}$ & NA \\
\hline modu-4-ne a & 351 & $\begin{array}{l}\text { miRNA-106b, - } \\
107,-125 b,-148 b \text {, } \\
-93\end{array}$ & $\begin{array}{l}\text { GO:0006955 immune response }(1.23 \mathrm{e}-20) \\
\text { GO:0006952 defense response }(1.88 \mathrm{e}-16) \\
\text { GO:0006954 inflammatory response }(2.02 \mathrm{e}-12) \\
\text { GO:0009611 response to wounding }(2.54 \mathrm{e}-12) \\
\text { GO:0002684 positive regulation of immune system process }(4.66 \mathrm{e}-10)\end{array}$ \\
\hline modu-4-ps & 191 & $\begin{array}{l}\text { miRNA-106b, - } \\
107,-125 b,-148 b \text {, } \\
-93\end{array}$ & $\begin{array}{l}\text { GO:0022403 cell cycle phase(8.60e-13) } \\
\text { GO:0007049 cell cycle(2.95e-12) } \\
\text { GO:0031981 nuclear lumen(1.53e-11) } \\
\text { GO:0044427 chromosomal part(2.39e-11) } \\
\text { GO:0022402 cell cycle process(9.06e-11) }\end{array}$ \\
\hline modu-5-ne ${ }^{a}$ & 494 & $\begin{array}{l}\text { miRNA-17, }-18 a,- \\
18 b,-20 a,-20 b\end{array}$ & $\begin{array}{l}\text { GO:0006955 immune response(1.58e-25) } \\
\text { GO:0006952 defense response(1.50e-20) } \\
\text { GO:0006954 inflammatory response(7.10e-16) } \\
\text { GO:0009611 response to wounding }(7.18 \mathrm{e}-14) \\
\text { GO:0045087 innate immune response(5.35e-12) }\end{array}$ \\
\hline modu-5-ps & 403 & $\begin{array}{l}\text { miRNA-17, -18a, - } \\
18 b,-20 a,-20 b\end{array}$ & $\begin{array}{l}\text { GO:0022403 cell cycle phase(3.65e-49) } \\
\text { GO:0000279 M phase(7.65e-47) } \\
\text { GO:0007049 cell cycle(1.93e-45) } \\
\text { GO:0022402 cell cycle process(1.33e-42) } \\
\text { GO:0000278 mitotic cell cycle(1.66e-38) }\end{array}$ \\
\hline
\end{tabular}

${ }^{a}$ semi-canonical regulatory module (semi-CRM). ${ }^{b} \mathrm{BH}$-adjusted $\mathrm{p}$-value. 
Table 2 The summary of the validation cohorts (datasets)

\begin{tabular}{lllcccl}
\hline Cohort & Dataset & Platform & \# Sample & follow-up (m) & (\%) & Reference \\
\hline Bentink & E.MTAB.386 & III. HumanRef-8v2 & 129 & 55 & 43 & (Bentink et al., 2012) \\
Bonome & GSE26712 & Affy U133a & 195 & 90 & 30 & (Bonome et al., 2008) \\
Crijns & GSE13876 & Aperon Human v3 & 157 & 72 & 28 & (Crijns et al., 2009) \\
Tothill & GSE9891 & Affy U133 Plus 2.0 & 285 & 36 & 59 & (Tothill et al., 2008) \\
Yoshihara & GSE32062 & Agilent G4112a & 260 & 56 & 53 & (Yoshihara et al., 2012) \\
\hline
\end{tabular}

